\title{
Jovian Cosmic-Ray Protons in the Heliosphere: Constraints by Cassini Observations
}

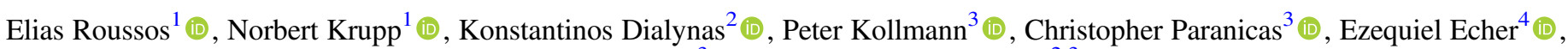 \\ Donald G. Mitchell ${ }^{3}$, and Stamatios M. Krimigis ${ }^{2,3}$ (i) \\ ${ }^{1}$ Max Planck Institute for Solar System Research, Justus-von-Liebig-Weg 3, D-37077, Goettingen, Germany; roussos@mps.mpg.de \\ ${ }^{2}$ Office of Space Research and Technology, Academy of Athens, Athens, Greece \\ ${ }^{3}$ Johns Hopkins University Applied Physics Laboratory, 11100 Johns Hopkins Road, Laurel, MD 20723-6099, USA \\ ${ }^{4}$ National Institute for Space Research (INPE), Sao Jose dos Campos, SP, Brazil \\ Received 2018 November 20; revised 2018 December 20; accepted 2018 December 28; published 2019 February 4
}

\begin{abstract}
Measurements of $>82 \mathrm{MeV}$ Galactic cosmic-ray (GCR) protons at Earth indicate that they may be mixed with protons that leak into the heliosphere from Jupiter's magnetosphere (Jovian cosmic-ray protons (JCRPs)). A $\sim 400$ day periodicity in these proton fluxes, which is similar to the synodic period between Jupiter and Earth, and an excess proton flux observed when Jupiter and Earth can be connected through the interplanetary magnetic field were the basis for this claim. Using nearly $13 \mathrm{yr}$ of GCR measurements at Saturn with Cassini's Magnetosphere Imaging Instrument, we show that the $\sim 400$ day periodicity is also present in $\gtrsim 100 \mathrm{MeV}$ protons at $\sim 9.6$ au, although the synodic period between Saturn and Jupiter is $\sim 20 \mathrm{yr}$. We also find that the features responsible for this periodicity were convected from 1 au to Saturn's distance with the solar wind velocity. Their origin is therefore heliospheric, not Jovian. We attribute these features to quasi-biennial oscillations, observed in the solar magnetic field and various heliospheric indices. This finding indicates that fluxes of JCRPs at 1 au, if present, are considerably overestimated, because the signal originally attributed to them represents the amplitude of the $\sim 400$ day periodic GCR oscillation. This oscillation has to be subtracted before the resulting proton GCR flux residuals are analyzed in the context of a possible Jovian source. A confirmation of the presence of JCRPs over extended regions in the heliosphere and a constraint on their fractional abundance in GCR spectra may therefore require further validation and analysis, and several options are proposed for this purpose.
\end{abstract}

Key words: cosmic rays - planets and satellites: gaseous planets - Sun: heliosphere

\section{Introduction}

The heliosphere is filled with charged particles covering a wide spectrum of energies. At the high-energy end of the spectrum, particles have a variety of sources, both internal and external to the heliosphere.

Galactic cosmic rays (GCRs) are charged particles of an external astrophysical origin and contain protons, heavier ions, and electrons with energies extending well above the $\mathrm{GeV}$ range (Blasi 2013). The fluxes of GCRs are dominated by protons and helium at the energy range of interest for the present study $(>80 \mathrm{MeV})$, and GCRs enter the heliosphere with an efficiency that depends on their energy and the phase of the $11 \mathrm{yr}$ solar cycle (Heber et al. 2006; Adriani et al. 2013; Jokipii 2013; Potgieter 2013, 2017). Solar and heliospheric sources add to the GCR population. They comprise solar energetic particles (SEPs), generated at solar flares; interplanetary coronal mass ejection (ICME) and corotating interaction region (CIR) shocks (Reames 1999; Cane 2000); and anomalous cosmic rays (ACRs), which are heavy ions accelerated up to several $\mathrm{GeV}$ somewhere at the outer edge of the heliosphere (Giacalone et al. 2012).

Magnetized planets can also supply the heliosphere with energetic particles. These particles get accelerated within their magnetospheres and have ways to escape into interplanetary space. Relativistic electrons from Jupiter that have been observed throughout the inner heliosphere are the most characteristic example for this case (Teegarden et al. 1974; Krimigis et al. 1975; Ferreira et al. 2003; Heber et al. 2007). When a spacecraft is connected to Jupiter through interplanetary magnetic field (IMF) lines, fluxes of $<30 \mathrm{MeV}$ Jovian electrons dominate over those of GCR electrons (Nndanganeni \& Potgieter 2018; Vogt et al. 2018), even though Jupiter is a point source in the heliosphere. Energetic ions of planetary origin and with subrelativistic energies $(<5 \mathrm{MeV})$ have been observed in the heliosphere upstream of magnetospheric bow shocks (Krimigis et al. 2009) or, in the case of Jupiter, within about 1 au of its magnetosphere (Marhavilas et al. 2001; Anagnostopoulos et al. 2009). A few studies, which relied on ground-based measurements by neutron monitors, indicated that Jupiter may also supply the heliosphere with protons of energies up to several GeV (Pizzella \& Venditti 1973; Mitra et al. 1983), but the interpretation of these measurements has been debated (Nagashima \& Tatsuoka 1984). Here we refer to these protons as Jovian cosmic-ray protons (JCRPs).

These past JCRP searches received support from spacebased measurements that were recently published by Adriani et al. (2018) and Pizzella (2018). The authors analyzed $82 \mathrm{MeV}-48 \mathrm{GeV}$ GCR proton data obtained with the Payload for Antimatter Matter Exploration and Light-nuclei Astrophysics (PAMELA) experiment, a high-precision energetic particle detector that was operating in low Earth orbit aboard the Russian Resurs-DK1 between 2006 and 2016 (Picozza et al. 2007). These PAMELA measurements reveal a series of periodicities, among which are several between 350 and 500 days. As this range contains the synodic period between Earth and Jupiter ( 299 days), and since an excess proton flux was measured when the connection between Earth and Jupiter across the IMF was expected to be more favorable, it was proposed that the fluxes of the detected protons, which are dominated by GCRs, also contained JCRPs, with an estimated contribution that ranges between $1 \%$ and $4.8 \%$ of the average GCR flux, depending on the proton energy.

On the other hand, Adriani et al. (2018) commented that proton flux periodicities in the $350-500$ day range may also 


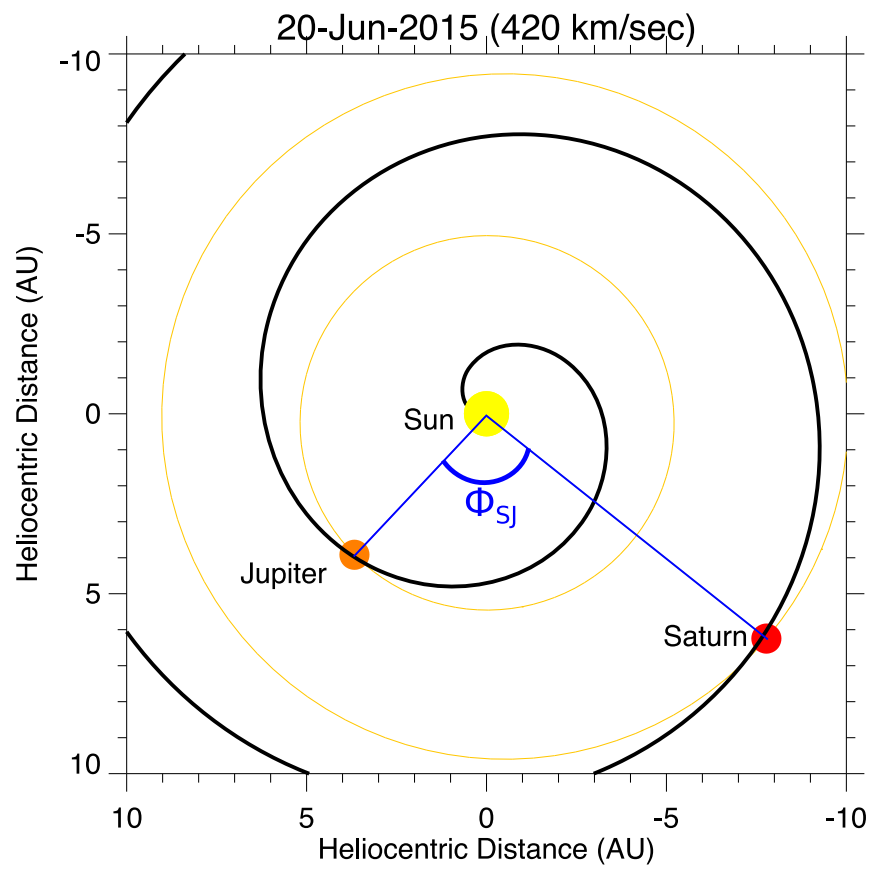

Figure 1. Jupiter and Saturn connected through the Parker spiral for 2015 June 20. The angle $\Phi_{\mathrm{SJ}}$ is the longitudinal separation of Jupiter and Saturn, as discussed in Section 3.3.

have a heliospheric origin but did not explore further whether that can be an alternative explanation to JCRPs. Such heliospheric "quasi-biennial oscillations" (QBOs) correspond to cyclic variations of the solar magnetic field with typical periods of 1.3 and $1.7 \mathrm{yr}$ (474 and 620 days) that get transmitted into the heliosphere and can modulate the fluxes of GCRs accordingly (Krivova \& Solanki 2002; Obridko \& Shelting 2007; Mandal et al. 2017). In addition, JCRP properties based on certain neutron monitor-based studies are not fully consistent with findings from PAMELA. For instance, Mitra et al. (1983) estimated that contributions of JCRPs at $>10 \mathrm{GeV}$ are $0.61 \%$ of the average GCR flux, several factors below what was estimated by Pizzella (2018), while in Mitra et al. (1982), the excess proton flux attributed to JCRPs was found to peak nearly $100^{\circ}$ away from the Earth-Jupiter longitudinal separation angle for which IMF connection between the two planets is expected to be optimal. For these reasons, the possibility that a link exists between energetic proton periodicities, excess fluxes observed with PAMELA, and JCRPs may benefit from further validation with independent data sets and/or methods.

The present study serves this purpose by extending the search for JCRPs at large heliospheric distances from Jupiter. We analyze the $13 \mathrm{yr}$ data set of Cassini's Magnetosphere Imaging Instrument (MIMI) at Saturn and the capability it offers to resolve time series of GCRs, as described in Roussos et al. (2018a). These MIMI measurements are among the few long-term GCR measurements at a relatively fixed heliocentric distance $(\sim 9.6 \mathrm{au})$, besides those obtained at $1 \mathrm{au}$. A key feature of our investigation is that the synodic period between Jupiter and Saturn is $\sim 19.9 \mathrm{yr}$, meaning that the 350-500 day periodicity seen at 1 au should be absent from the Cassini data set if it is caused primarily by JCRPs. In addition, because the Parker spiral winds up at least once by 9.6 au (Figure 1), the IMF equatorial projection at Saturn is nearly azimuthal (Jackman et al. 2008). That means that a connection between Cassini and Jupiter through the IMF may be possible over a large longitude range across Saturn's orbit. A strong JCRP signal may then be the source of frequent periods with qualitative differences between the GCR time series at Earth and the respective MIMI measurements at Saturn.

\section{Instrumentation}

The MIMI instrument (Krimigis et al. 2004) comprises three different detector systems: the Low Energy Magnetospheric Measurement System (LEMMS), CHarge Energy Mass Spectrometer (CHEMS), and Ion Neutral CAmera (INCA). We will use measurements only from LEMMS, a double-sided particle telescope that was designed to primarily measure protons between $20 \mathrm{keV}$ and $\sim 100 \mathrm{MeV}$ and electrons between $18 \mathrm{keV}$ and $\sim 10 \mathrm{MeV}$.

While LEMMS's intended energy coverage and geometry factors were suitable for magnetospheric studies, its highenergy channels received a notable signal from GCRs (Roussos et al. 2018a). That is because GCRs could penetrate LEMMS's shielding and access its detectors from a large range of directions. In addition, many channels that were designed to measure $\mathrm{MeV}$ electrons can also respond to $>100 \mathrm{MeV}$ protons. This proton response becomes dominant when fluxes of $\mathrm{MeV}$ electrons are negligible, as will be the case with the data we present here. Because of that, the proton energy coverage of LEMMS extends above $300 \mathrm{MeV}$, beyond the instrument's design capabilities (Roussos et al. 2018b).

The GCR signal in LEMMS can be directly isolated for times that magnetospheric $\mathrm{MeV}$ electron or ion fluxes were below LEMMS's detection threshold, essentially when Cassini was away from Saturn's radiation belts (Appendix A). Because crossings of the radiation belts were brief (typically lasting less than $12 \mathrm{hr}$ ), and since GCRs can easily penetrate into the outer and middle magnetosphere of Saturn (Kotova et al. 2019), monitoring of GCRs with LEMMS was nearly continuous for $13 \mathrm{yr}$, even within the boundaries of Saturn's magnetosphere.

We use data from three LEMMS channels: P9, E6, and E7. All of these have double-species responses ( $\mathrm{MeV}$ electrons and protons; Figure 2), but only the proton response is relevant because there are not enough $\mathrm{MeV}$ electrons to yield significant count rates of the radiation belts, as considered here (Appendix A). The omnidirectional proton response functions of these channels are shown in Figure 2(a), and they are based on simulations described in Roussos et al. (2018b). As we will show later (Section 3.2), these geometry factors overestimate the detection efficiency of GCR protons, mostly because they were calculated without taking into consideration the shielding of LEMMS by the Cassini spacecraft.

Channels P9 and E6 are used due to their sensitivity to instrument penetrating, GCR protons, giving GCR signals between 0.9 and 2.6 counts $\mathrm{s}^{-1}$. These values are orders of magnitude above the single-count level when a daily averaging is applied to the data (Figure 3(a)). Sources of noise for E6 and P9 are gamma rays from Cassini's radioisotope thermoelectric generators (RTGs). This noise level is difficult to determine, but it is likely below 0.1 counts $\mathrm{s}^{-1}$. Channel E7 has a lower count rate but a better rejection of sideways-penetrating protons (Figure 3(b)) and no RTG noise. Any systematic errors in E7's response function from the fact that spacecraft shielding was not modeled are less important than those for P9 and E6, as will be demonstrated in 

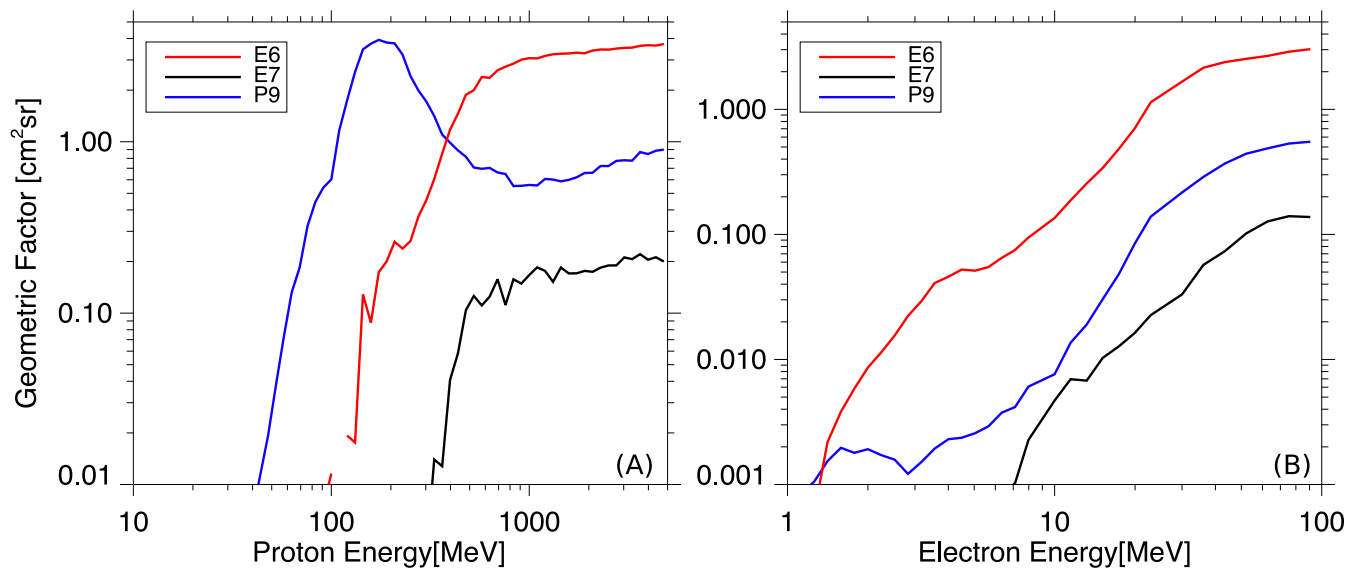

Figure 2. Omnidirectional response functions of LEMMS channels E6, E7, and P9 to protons and electrons (panels (a) and (b), respectively) based on the simulations described in Roussos et al. (2018b). Electron response functions are used in calculations discussed in Appendix A.
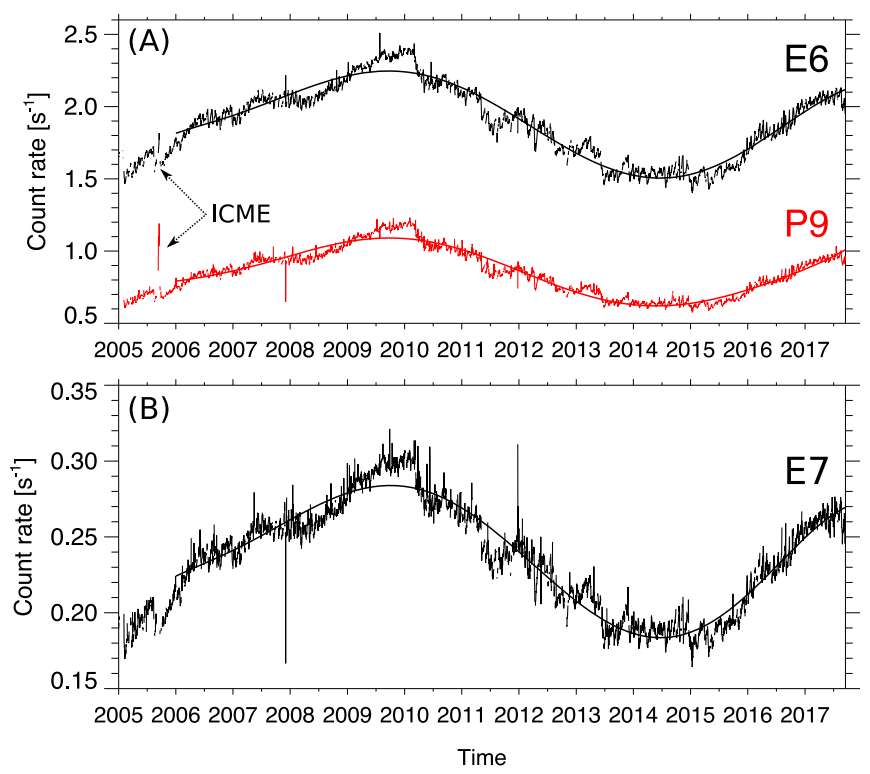

Figure 3. Time series of LEMMS measurements from channels E6, P9 (panel (a)), and E7 (panel (b)). All data are averaged daily in order to enhance their signal-to-noise ratio. Periods of radiation belt crossings are excluded in order to isolate GCR counts (Roussos et al. 2018a). Several residual spikes visible in the data set that are due to instrumental effects and were not removed by the median filter applied have no impact on our analysis. The spike in the second half of 2005 (panel (a)) is due to a strong ICME event (Roussos et al. 2008). Smooth curves are seventh-order polynomial fits to the data.

Section 3.2. In addition, its lower energy threshold at $\sim 300 \mathrm{MeV}$ makes it insensitive to SEPs superimposed on the GCR variations, even during strong ICME events.

The data we present are averaged daily and cover the time period between day 32/2005 and the end of the Cassini mission (day 258/2017). The first date is after the time when the scan platform of LEMMS stopped operating and the atmospheric probe Huygens was released toward Saturn's moon Titan (day 360/ 2004), with both events slightly affecting the shielding of LEMMS from GCRs. Additionally, a series of changes in the energy thresholds of LEMMS's channels before Saturn Orbit Insertion (day 183/2004) are not yet considered in the calibration curves of Figure 2. Additional details of the processing of the daily averaged data are given in Roussos et al. (2018a).

\section{Data Analysis}

\subsection{Periodicities in the LEMMS Signal}

Figure 3 shows the data from the three aforementioned channels of LEMMS. All show the well-understood 11 yr solar cycle periodicity of GCRs. At short timescales, a solar rotation periodicity of $\sim 26$ days and with an amplitude of $\sim 10 \%$ of each channel's signal, which is attributed to CIRs, is resolvable "by eye" in certain periods (e.g., 2006-2009, 2016-2017), especially in channels E6 and P9. To further enhance the signal of additional periodicities, we apply the following procedure.

At first, we decided to exclude the data before 2006 due to the occurrence of a strong ICME that caused a long-duration Forbush decrease during the second half of 2005 in all three channels. A spike in channels E6 and P9 is the SEP signature of this previously studied ICME (Roussos et al. 2008). Other ICMEs and their associated Forbush decreases are much shorter in duration and intensity, at least for these three LEMMS channels, and do not affect our analysis (Roussos et al. 2018a).

A second step involves a fitting of the GCR profiles with simple polynomials in order to remove the solar cycle trend and enhance the signal of secondary variations and periodicities, as was also done in Adriani et al. (2018) and Pizzella (2018). The fits, using seventh-order polynomials, are the smooth curves overplotted on the data shown in Figure 3.

We then apply a Lomb-Scargle analysis to the residuals of each of the three LEMMS channels. The resulting periodograms are shown in Figure 4(a). Several peaks can be resolved, with the most characteristic one around 26 days. Of interest to this study is a peak at $400 \pm 100$ days, in the range reported by Adriani et al. (2018).

We have also repeated this analysis in a time-resolved manner to investigate whether this 400 day period is persistent. We separated the LEMMS time series into three time periods.

1. Declining phase of solar cycle 23 (23d): days 001/ 2006-180/2009.

2. Ascending phase of solar cycle 24 (24a): days 180/ 2010-180/2014.

3. Declining phase of solar cycle 24 (24d): days 180/ 2014-258/2017.

Detrending the long-term GCR profile for each time period was done with third-order polynomials. Between phases $23 \mathrm{~d}$ 

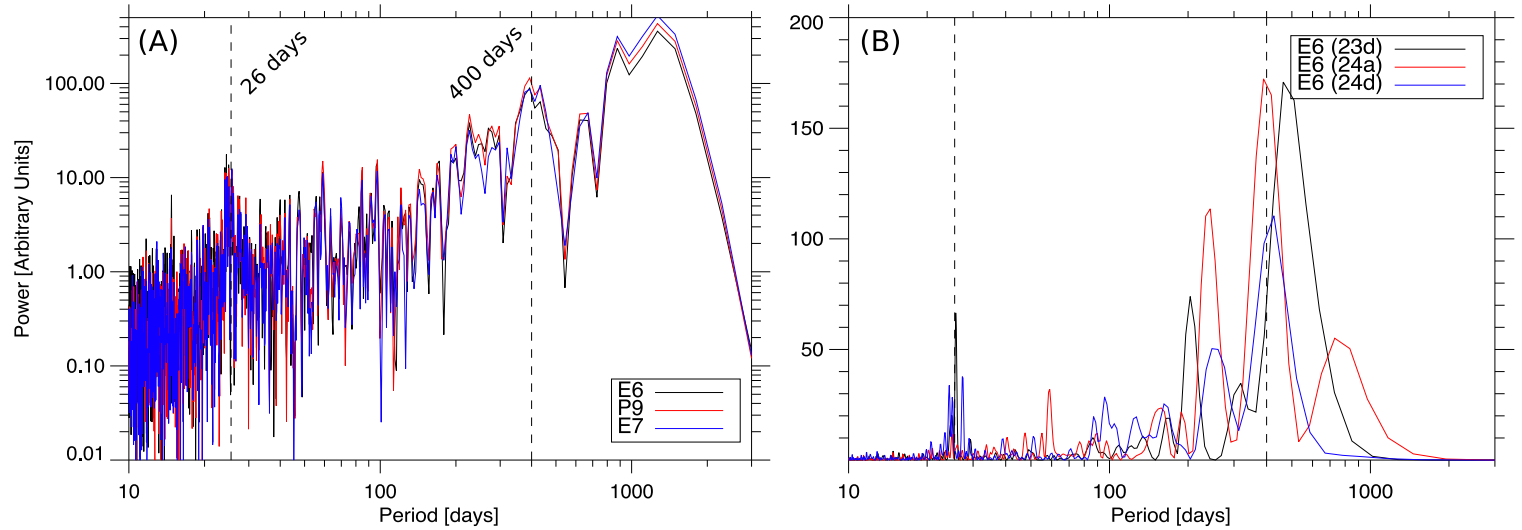

Figure 4. Panel (a): Lomb-Scargle periodogram on the LEMMS channel residual signal from 2006 January until the end of the Cassini mission. Panel (b): LombScargle periodogram on LEMMS channel E6 for different time periods, as defined in the text. Two periods are highlighted (26 and 400 days), although several more peaks are visible. The second panel is plotted in linear scale to better highlight the 400 day period. Similar results are obtained if a fast Fourier transform is applied to the time series or, in the case of panel (b), if a different LEMMS channel is used. The peak around 1000 days does not appear in panel (b), due to the shorter time ranges analyzed.

and 24a, we excluded $1 \mathrm{yr}$ of measurements around the solar minimum for a better comparison with Adriani et al. (2018) and Pizzella (2018). We also note that analysis of PAMELA data for JCRPs has gone up to day 243/2014.

Figure 4(b) shows the results of the time-resolved analysis based on the E6 time series. Some effects of splitting the LEMMS time series are seen in the 26 day peak, which is prominent mostly for the two declining solar cycle phases $(23 \mathrm{~d}$ and 24d), when CIRs are expected to be more regular (Jackman et al. 2008; Zhang et al. 2008). The peak around $400 \pm$ 100 days remains strong, even though it is broader due to the smaller sample used for the three time periods.

\subsection{Comparison of GCR Variations at Earth and Saturn}

In order to better understand what part of the GCR proton spectrum dominates the LEMMS observations shown in Figure 3 and compare them with GCR measurements at Earth, we reconstruct the LEMMS count rates using the force-field approximation that offers a practical description of the GCR spectra in the inner heliosphere (Gleeson \& Axford 1968; Vainio et al. 2009). Despite its limitations, this approximation represents the shape of the GCR proton energy spectrum with good accuracy at $<20 \mathrm{au}$, even at energies below $100 \mathrm{MeV}$ (Caballero-Lopez \& Moraal 2004). According to the force-field formalism, the GCR differential energy proton spectrum, $j(t, T)$, is described as

$$
j(t, T)=j_{\mathrm{LIS}}(T+\Phi(t)) \frac{E^{2}-T_{o}^{2}}{(E+\Phi(t))^{2}-T_{o}^{2}},
$$

with the GCR spectrum of the local interstellar medium $\left(j_{\text {LIS }}\right)$ given as

$$
j_{\text {LIS }}=2.7 \times 10^{3} \frac{T^{1.12}}{\beta^{2}}\left(\frac{T+0.67}{1.67}\right)^{-3.93}\left(m^{2} \mathrm{~s} \mathrm{sr} \mathrm{GeV}\right)^{-1} \text {. }
$$

The spectrum is parameterized by the modulation potential, $\phi[\mathrm{GV}]$. When expressed in terms of energy $(\Phi[\mathrm{GeV}]=\mathrm{e} \phi$, with $e$ the electron charge), it corresponds to the average energy lost by GCR protons inside the heliosphere. Time series of $\Phi$ have been calculated at 1 au and up to the end of 2016 through the combined analysis of neutron-monitor data and measurements of GCR spectra by PAMELA and are provided, together with Equations (1) and (2), in Usoskin et al. (2017). Other parameters in the two equations are the total proton energy $\left(E=T+T_{o}[\mathrm{GeV}]\right)$, the rest mass energy of the proton $\left(T_{o}=0.938 \mathrm{GeV}\right)$, its kinetic energy $(T[\mathrm{GeV}])$, and the ratio of the proton speed to the speed of light $(\beta=v / c)$.

In order to reconstruct the count rates of a LEMMS channel (i), we convolve Equation (1) with the response function of each channel, $G_{i}(T)$ (Figure 2), and obtain a simulated rate, $R_{\mathrm{S}, i}(t)$ :

$$
R_{\mathrm{S}, i}(t)=\frac{4 \pi \cdot 1.279}{k_{i}} \int_{T_{\min }}^{50 \mathrm{GeV}} j(t+\Delta t, T) G_{i}(T) d T .
$$

The reconstruction assumes that $\Phi$ at Saturn's distance is reduced by $\sim 10 \%$ with respect to its value at 1 au (CaballeroLopez \& Moraal 2004) and that the GCR influx on LEMMS is isotropic $(4 \pi)$. The factor 1.279 accounts for an $\sim 3.1 \% \mathrm{au}^{-1}$ radial GCR flux gradient (Gieseler \& Heber 2016) in the proton energy range of $\sim 0.1-1 \mathrm{GeV}$, where contribution to the LEMMS channels' rates is the highest. The minimum energy, $T_{\min }$, is the lowest energy where a channel has a response. The constant $k_{i}$ is purely empirical and accounts for any source of uncertainties and systematic errors in our assumptions and the input parameters of Equation (3). It scales $R_{\mathrm{S}, i}(t)$ such that it can be best compared with the measured LEMMS rates. The parameter $\Delta t$ introduces a time lag in the propagation of the GCR modulation from 1 au (where time series of $\Phi$ are given) to Saturn's orbit, where LEMMS data are obtained. It is determined by our analysis, as explained in the follow-up paragraphs.

Equation (3) is integrated up to $50 \mathrm{GeV}$. Since the simulated response functions go up to $5 \mathrm{GeV}$ (Figure 2), we extrapolate them linearly to higher energies. For this extrapolation, we have calculated the geometry factor of each channel at $50 \mathrm{GeV}$ as in Roussos et al. (2018b). The values for E6, P9, and E7 are 4.6, 2.7, and $0.3 \mathrm{~cm}^{2} \mathrm{sr}$, respectively. Beyond $50 \mathrm{GeV}$, the proton contribution to the count rate of each LEMMS channel is lower than $1 \%$.

Panels (a)-(c) of Figure 5 show that our method reconstructs the LEMMS count rate profiles successfully. The reconstruction not only reproduces the general trend and variability amplitude in the LEMMS rates but also captures medium-term dynamics (timescales of 50-100 days), such as the large Forbush decrease during the second half of 2005. 

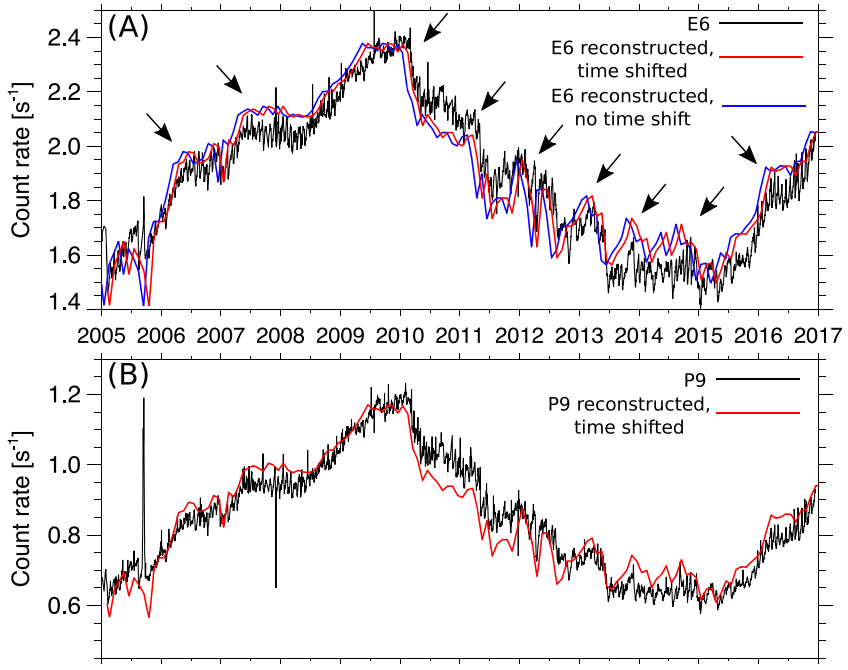

2005200620072008200920102011201220132014201520162017

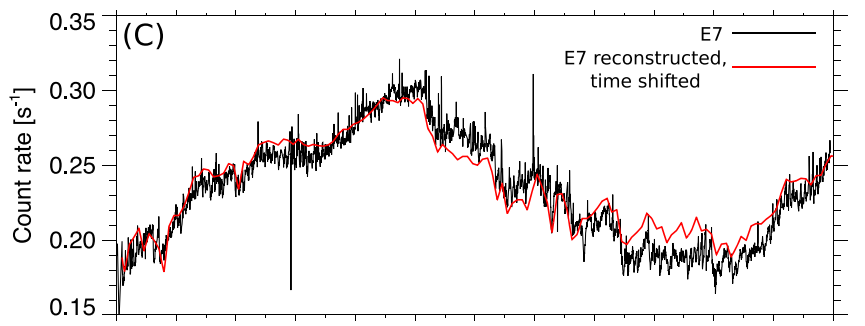

2005200620072008200920102011201220132014201520162017

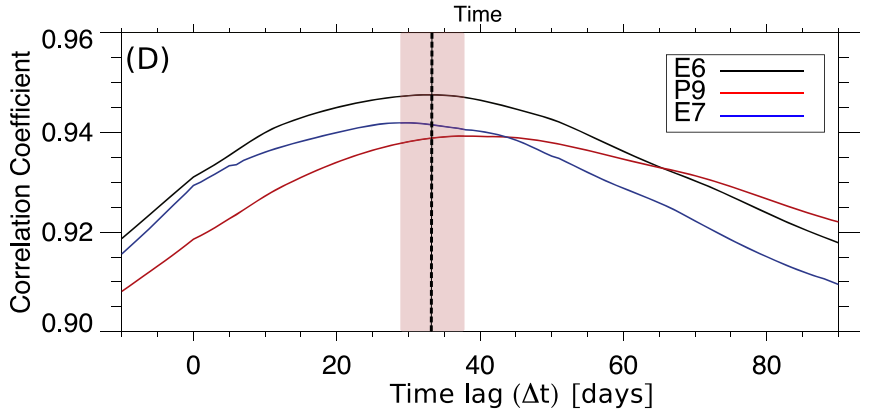

Figure 5. Panels (a)-(c): LEMMS time series for channels E6, P9, and E7 (black) and their reconstruction based on Equations (1)-(3) (red), including an average time shift of $\overline{\Delta t}=33$ days. Nonshifted data are shown in panel (a) for comparison (blue), together with arrows highlighting features responsible for the $400 \pm 100$ day periodicity. Panel (d): linear correlation coefficient between the measured and reconstructed signal as a function of the time lag, $\Delta t$. The dashed line and shaded area correspond to $\overline{\Delta t}=33 \pm 4$ days. The correlation coefficient is high even for values much different than $\overline{\Delta t}$ because this time lag is much shorter than the nearly $13 \mathrm{yr}$ duration of the LEMMS observations.

Table 1 lists reconstruction parameters. The values of $k_{i}$, which scale $R_{\mathrm{S}, i}(t)$ to the levels of the LEMMS rates, are between 3.0 and 6.9. Our approach therefore overestimates the LEMMS rates. Still, these $k_{i}$ values are rather small given that LEMMS was not designed to measure GCRs and that the GCR spectrum extends to extreme energies, meaning that systematic errors in $G_{i}(T)$ are integrated through Equation (3) over a very large energy range. If we assume that the excess count rate is dominated by a systematic error in $G_{i}(T)$, then the $k_{i}$ values indicate that the average $G_{i}(T)$ overestimation ranges between $6 \%$ and $14 \% \mathrm{GeV}^{-1}$, suggesting that the LEMMS response functions that we use (Figure 2) are sufficient for the analysis done here.

Panel (d) of Figure 5 shows the linear correlation coefficient between $R_{\mathrm{S}, i}(t)$ and the measured GCR profile by LEMMS as a
Table 1

Parameter Values in Equation (3) Used for the LEMMS Signal Reconstructions Shown in Figure 5

\begin{tabular}{lccc}
\hline \hline Parameter/Channel & E6 & P9 & E7 \\
\hline$k$ & $6.9 \pm 0.05$ & $5.65 \pm 0.05$ & $3.00 \pm 0.05$ \\
$\Delta t$ (days) & 33 & 37 & 30 \\
\hline
\end{tabular}

function of the time lag, $\Delta t$ (Equation (3)). Depending on the LEMMS channel, the correlation maximizes for $\Delta t$ between 30 and 37 days, with an average $\overline{\Delta t}=33 \pm 4$ days. This translates to an average solar wind velocity between Earth and Saturn of $431 \pm 52 \mathrm{~km} \mathrm{~s}^{-1}$, similar to the value of $430 \pm 54 \mathrm{~km} \mathrm{~s}^{-1}$ estimated by Echer (2018), based on in situ measurements of solar wind velocity with the Voyager and Pioneer spacecraft.

The effects of the time lag are demonstrated in panel (a) of Figure 5. The necessity for the time correction is most easily visible at the date of the peak count rates between mid-2009 and 2010 (solar minimum). Most important for our study is that the time shift improves the coincidence of a series of broad, transient maxima in $R_{\mathrm{S}, i}(t)$ with similar features seen by LEMMS. These maxima, indicated by arrows in panel (a), are responsible for the $400 \pm 100$ day periodicity reported by Adriani et al. (2018) and at $9.6 \mathrm{au}$, as identified through the analysis shown in Section 3.1. This observation suggests that these quasi-periodic features are convected from the inner toward the outer heliosphere with the solar wind velocity.

\subsection{Searching for an Excess Signal}

A possible contribution of JCRPs in the LEMMS rates can be revealed after we subtract from them the reconstructed count rate profiles and organize the residuals as a function of the angle $\Phi_{\mathrm{SJ}}=\Phi_{\mathrm{S}}-\Phi_{\mathrm{J}}$. This angle is the longitude separation of Saturn from Jupiter (measured counterclockwise) in a system corotating with the Sun-Jupiter line, as shown in Figure 1. Following Pizzella (2018), for Jupiter and Saturn to be connected through the IMF, the longitude separation $\left(\Phi_{\mathrm{SJ}}^{*}\right)$ should be

$$
\Phi_{\mathrm{SJ}}^{*}=\frac{0.9}{\gamma}\left(R_{\mathrm{S}}^{\gamma}-R_{\mathrm{J}}^{\gamma}\right) \frac{V_{\mathrm{sw}}}{400} .
$$

The solar wind velocity $\left(V_{\mathrm{sw}}\right)$ is given in $\mathrm{km} \mathrm{s}^{-1}$, and the heliocentric distances of Saturn and Jupiter $\left(R_{\mathrm{S}}, R_{\mathrm{J}}\right)$ are given in au. The constant $\gamma$ is linked to the Parker spiral angle, $\Phi_{B}$, through $\operatorname{Tan}\left(\Phi_{B}\right) \sim R^{\gamma}$ and takes values between 0.37 and 0.54 , as summarized in Pizzella (2018). Taking into account this uncertainty in $\gamma$, variations in the solar wind velocity (Section 3.2), and the eccentricity of Jupiter's and Saturn's orbits, we estimate that the angular range at which the two planets may become magnetically connected should be $50^{\circ}<\Phi_{\text {SJ }}^{*}<130^{\circ}$. Angles of $55^{\circ}-130^{\circ}$ were covered after 2014 mid-June and until the end of the mission, while the remaining angular range was not sampled by Cassini. Due to the variability discussed above, connection of Cassini with Jupiter would likely be sporadic rather than continuous during the periods that $\Phi_{\mathrm{SJ}}^{*}$ is covered, such that JCRPs do not modify the long-term, solar cycle trend of GCRs. We can then subtract this long-term trend and look for JCRPs in the count rate residuals.

Plots of residual count rates as a function of $\Phi_{\mathrm{SJ}}$ for channel E7 are shown in Figure 6 . The $\Phi_{\mathrm{SJ}}^{*}$ range, where we would 
Table 2

Mean Residual Count Rate $(r)$ and Standard Deviation $(\sigma)$ for the Angular Interval $55^{\circ}<\Phi_{\mathrm{SJ}}<130^{\circ}$, Where Jupiter and Saturn May Get Connected across the IMF $\left(r_{1}, \sigma_{1}\right)$, and for the Rest of the Angular Range Covered during the Cassini Mission $\left(\Phi_{\mathrm{SJ}}>130^{\circ}\right)\left(r_{2}, \sigma_{2}\right)$

\begin{tabular}{|c|c|c|c|c|c|c|}
\hline \multirow[t]{2}{*}{ Channel } & \multicolumn{2}{|c|}{$50^{\circ}<\Phi_{\mathrm{SJ}}<130^{\circ}$} & \multicolumn{2}{|c|}{$\Phi_{\mathrm{SJ}}>130^{\circ}$} & \multicolumn{2}{|c|}{ Difference } \\
\hline & Residuals $\left(\mathrm{s}^{-1}\right)$ & $\sigma\left(\mathrm{s}^{-1}\right)$ & Residuals $\left(\mathrm{s}^{-1}\right)$ & $\sigma\left(\mathrm{s}^{-1}\right)$ & Residuals $\left(\mathrm{s}^{-1}\right)$ & $\overline{\sigma\left(\mathrm{s}^{-1}\right)}$ \\
\hline E6 & $-2 \times 10^{-4}$ & $5 \times 10^{-2}$ & $2 \times 10^{-4}$ & $7 \times 10^{-2}$ & $4 \times 10^{-4}$ & $9 \times 10^{-2}$ \\
\hline
\end{tabular}

Note. The difference for the residuals is $r=r_{1}-r_{2}$ and $\sigma=\sqrt{\sigma_{1}^{2}+\sigma_{2}^{2}}$.

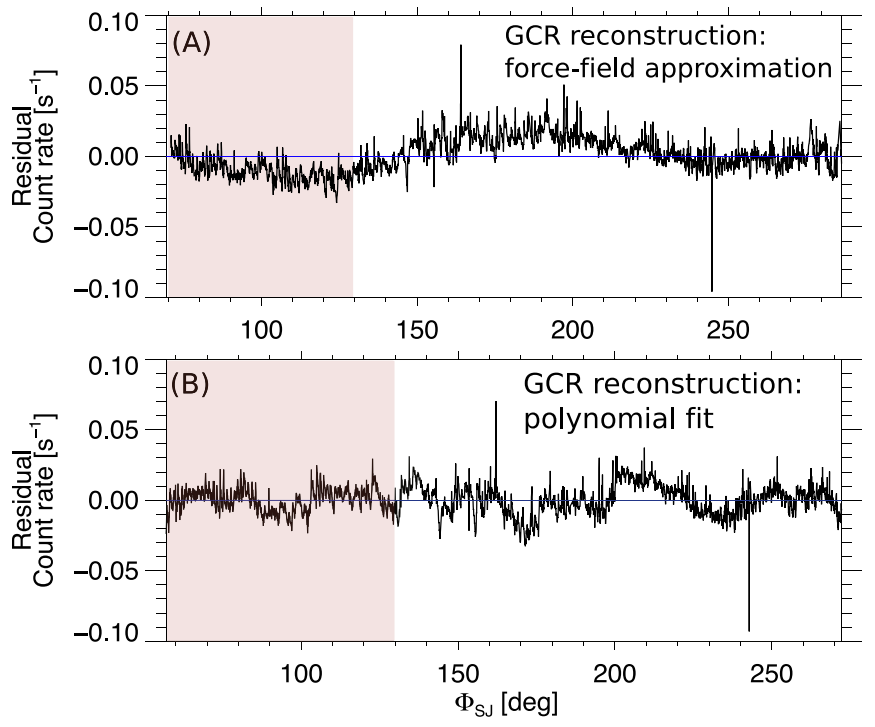

Figure 6. Residual LEMMS count rate after subtracting a physics-based and an empirical representation of the LEMMS signal (panels (a) and (b), respectively). The shaded areas correspond to the angular range at which Jupiter and Saturn may become connected across the IMF.

expect higher count rates if JCRPs reach Saturn, is shaded. The residuals in Figure 6(a) were obtained after subtracting force field-based reconstruction of the LEMMS signal, as discussed in Section 3.2 (Equations (1)-(3)). The residuals in Figure 6(b) were calculated by subtracting the seventh-order polynomial (Figure 3(b)). The first method (panel (a)) captures both medium- and long-term dynamics but has various other sources of uncertainties in the input parameters, such as LEMMS's response functions, time-dependent GCR radial gradients (Caballero-Lopez \& Moraal 2004), etc. The second method (panel (b)) removes only the long-term, solar cycle trend of the signal, which is why medium-term dynamics remain. The two methods can be seen as complementary.

None of the two methods hints at a surplus signal from JCRPs. On the contrary, the force field-based reconstruction (Figure 3(a)) would indicate a deficiency of GCRs at the $\Phi_{\mathrm{SJ}}^{*}$ interval. We believe that this systematic deficiency is likely due to uncertainties and systematic errors in the reconstruction method, as mentioned above, because the residual profile varies smoothly as a function of $\Phi_{\mathrm{SJ}}$. For the case that the long-term trend of the GCR signal is described through a polynomial function (Figure 3(b)), we compared the mean residual count rates for times in and out of the $\Phi_{\mathrm{SJ}}^{*}$ angular interval (Table 2). Since for each channel, the mean values in the two angular intervals are comparable, and their difference is much lower than their corresponding standard deviation, we conclude that there is no statistically resolvable surplus signal from JCRPs that can be clearly separated from the overall LEMMS count rate variations. We note that these results apply even if we detrend the LEMMS count rate profiles with polynomials of different order or in a time-resolved manner.

\section{Discussion and Conclusions}

Nearly $13 \mathrm{yr}$ of GCR proton data captured by Cassini's MIMI/LEMMS sensor at Saturn were analyzed in order to evaluate whether GCRs in the heliosphere are mixed with $>82 \mathrm{MeV}$ protons from Jupiter (JCRPs), as has been suggested from Earth-based observations (Adriani et al. 2018; Pizzella 2018).

Our principal result is that the bulk of the $\sim 400$ day periodic signal seen in GCR protons at 1 au has a heliospheric and not a Jovian origin. One reason is that the same periodicity is seen in LEMMS measurements at $9.6 \mathrm{au}$, where the synodic period between Jupiter and Saturn is 19.9 yr. In addition, a force fieldbased reconstruction of the LEMMS signal shows that the quasi-periodic features that are responsible for the $\sim 400$ day cycle at Earth also occur at Saturn with a time lag of $\sim 33$ days, meaning that they get convected from 1 to 9.6 au with the solar wind velocity.

These Cassini observations favor the alternative interpretation for the $\sim 400$ day proton periodicities discussed by Adriani et al. (2018): these are QBOs of the heliospheric magnetic field, which transmit their modulation to GCR fluxes. The periodicity peak at $\sim 400$ days seen with Cassini is slightly shorter than the closest, most typical QBO period of $1.3 \mathrm{yr}$ (474 days) that is reported in the literature (Mandal et al. 2017). In addition, Obridko \& Shelting (2007) recognized that 1.3 yr QBOs occur preferentially at the declining phase of solar cycles or during solar maxima, while we see them recurring at all solar cycle phases (Figure 4(b)). Those differences, which may be due to the evolution of heliospheric oscillations from the innermost heliosphere to Saturn's distance and/or a characteristic specific to solar cycles 23 and 24, are not against the interpretation of these features as QBOs. Additional periodicities, e.g., at $~ 250$, $\sim 650, \sim 850$, and $\gtrsim 1000$ days, that are seen with LEMMS but not explicitly discussed here (Figure 4(a)) may be relevant to other peaks in the QBO periodicity spectrum (see review by Bazilevskaya et al. 2014). LEMMS measurements are therefore significant for studying how QBOs propagate from the Sun and into the heliosphere and how they evolve across different solar cycles.

We now explain why this heliospheric GCR flux oscillation results in some misleading coincidences that may affect the search for JCRPs at $1 \mathrm{au}$. Since the QBO period is similar to the synodic period of Jupiter seen from Earth, its profile as a function of the Earth-Jupiter longitudinal separation $\left(\Phi_{\mathrm{EJ}}\right.$, defined analogously 

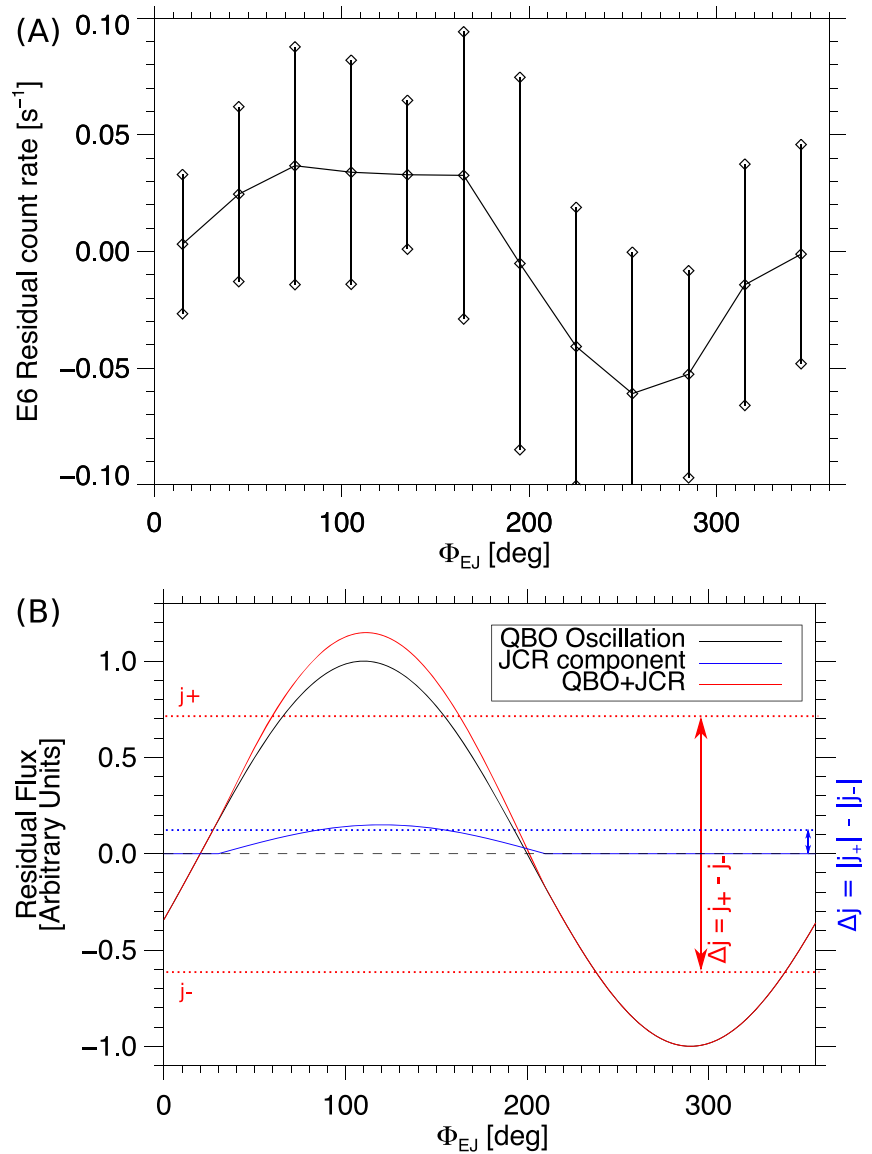

Figure 7. Panel (a): LEMMS E6 channel residual count rates for the declining phase of the solar minimum (23d), after subtraction of a third-order polynomial describing the long-term solar cycle GCR variability (Figure 3, Section 3.1), organized as a function of the Earth-Jupiter longitudinal separation, $\Phi_{\mathrm{EJ}}$, similar to Figure 4 of Adriani et al. (2018). Panel (b): schematic illustration of a heliospheric, 400 day GCR flux oscillation (black), superimposed on a weak JCRP component (blue), organized as a function of $\Phi_{\mathrm{EJ}}$. The parameters $j_{+}, j_{-}$ are the average fluxes of the positive and negative phases of the sinusoidal flux pulse.

as $\Phi_{\mathrm{SJ}}$ in Figure 1) would be quasi-sinusoidal. To illustrate this, we organize the LEMMS residual count rates (after subtracting the solar cycle trend) as a function of $\left(\Phi_{\mathrm{EJ}}\right)$, even if this angle is physically meaningless for proton measurements at Saturn (Figure 7(a)). Despite that, proton rates show a clear sinusoidal behavior. Another coincidence is that the phase of the 400 day oscillation is such that the count rate peaks at $\Phi_{\mathrm{EJ}}$ of $\sim 100^{\circ}-140^{\circ}$, the angular range where the Jupiter-Earth IMF connection is expected to be optimal (Pizzella 2018).

A similar picture to Figure 7(a) has been obtained from the PAMELA measurements at 1 au (Figure 4 of Adriani et al. 2018). For these measurements, however, the assumption was that the sinusoidal behavior of the residual fluxes was due to JCRPs. The same assumption is effectively retained in Pizzella (2018), since the 400 day heliospheric oscillation was not subtracted from the GCR time series. The implications are explained through the schematic of Figure 7(b), where a sinusoidal heliospheric proton flux oscillation as a function of $\Phi_{\mathrm{EJ}}$ is superimposed on a flux enhancement from JCRPs. Both profiles peak at a similar $\Phi_{\mathrm{EJ}}$ range, as discussed in the context of Figure 7(A). What an instrument would observe is the sum of the two proton flux profiles and without a direct option to distinguish the different contributions. It is the assumption about which of the two contributions dominates that leads to significantly different interpretations about the flux levels of JCRPs.

If it is assumed that the pulse has a Jovian origin, the average excess JCRP flux becomes the difference between the average fluxes in the positive and negative phases of the sinusoidal signal, or $\Delta j=j_{+}-j_{-}$. Since $j_{-}<0$ and $j_{+} \approx\left|j_{-}\right|$(Figure 4 of Adriani et al. 2018 and Figure $7(\mathrm{a})$ ), then $\approx 2 j_{+}$, that is, twice the mean amplitude of the residual flux oscillation. Analysis of Cassini data, however, shows that it is the heliospheric QBO component that dominates the oscillation, meaning that a flux of $\sim 2 j_{+}$corresponds to the mean amplitude of the QBO pulses. Because of that, it is not sufficient to subtract only the solar cycle trend in order to calculate flux residuals and associate them with JCRPs, as done in Adriani et al. (2018) and Pizzella (2018); trends due to QBOs should also be subtracted.

If only the solar cycle trend is removed, a possible contribution from JCRPs would be no larger than $\Delta j=\left|j_{+}\right|-\left|j_{-}\right| \ll 2 j_{+}$ (Figure 7(b)). Since Figure 4 of Adriani et al. (2018) shows that the positive and negative phases of the sinusoidal residual flux profiles have similar amplitudes, we expect that the abundance of JCRPs at $1 \mathrm{au}$ is considerably overestimated. Consequently, a direct detection of JCRPs with LEMMS at $9.6 \mathrm{au}$ is also not possible (Appendix B). Further searches for JCRPs at large heliocentric distances from Jupiter and a characterization of their spectral properties would require a reevaluation of the Earthbased, GCR proton measurements, as well as analysis of additional data sets. Several data sets and methods can be considered for this purpose.

A similar type of analysis, as presented in Adriani et al. (2018), Pizzella (2018), and here, can be performed through GCR measurements that are now available at Mars (e.g., Hassler et al. 2014; Semkova et al. 2018). At Earth, a possible excess signal in GCR protons from JCRPs should be correlated in time with detections of Jovian relativistic electrons, which are easier to recognize. Heavy ions characteristic of the Jovian magnetospheric composition (oxygen, sulfur) may be reliable tracers of JCRPs, since fluxes of GCR heavy ions are very low (Blasi 2013). The long-term data set (1997-present) from the Cosmic-Ray Composition Instrument (CRIS; Stone et al. 1998) on the Advanced Composition Explorer mission may well be the best for this task.

Critical for evaluating the presence of JCRPs are observations near and within Jupiter's magnetosphere, which can inform us about the efficiency proton and ion acceleration in that system and what the spectrum of the particles escaping into the heliosphere is. The search for very high energy protons and heavy ions through measurements near Jupiter by Voyager (Krimigis et al. 1977; Stilwell et al. 1979), Ulysses (Simpson et al. 1992), Juno (Mauk et al. 2013; Becker et al. 2017), and Galileo (Garrard et al. 1992; Williams et al. 1992) could constrain the spectrum of possible JCRPs escaping from the planet's magnetosphere and allow for a comparison with observations at different heliocentric distances, similar to Vogt et al. (2018). Within the Jovian magnetosphere, protons and ions between several $\mathrm{MeV} \mathrm{n}^{-1}$ and up to $\sim 100 \mathrm{MeV} \mathrm{n}^{-1}$ have been observed in the planet's radiation belts, at an acceleration region in Jupiter's middle and outer magnetosphere, or on highlatitude field lines possibly mapping to the auroral region 
(Zhang et al. 1995; Fischer et al. 1996; Anglin et al. 1997; Selesnick et al. (2001).

No measurements at proton or ion energies at $\gtrsim 100 \mathrm{MeV}$ exist at Jupiter, but they may become available through the New Horizons spacecraft, which flew by Jupiter and can resolve GCRs or very energetic ions in the same way we do with Cassini here (Hill et al. 2018). Further calibrations of Cassini's MIMI/LEMMS would extend the GCR data set back to 1999 , including the Jupiter flyby (Krupp et al. 2002). Despite the lack of measurements at $\gtrsim 100 \mathrm{MeV}$, we know that at least the cosmic-ray albedo neutron decay process (CRAND; Dragt et al. 1966) can supply protons well above energies of $1 \mathrm{GeV}$. Even though its efficiency is likely lower at Jupiter compared to Earth and Saturn (Spergel 1977), it may still dominate the production of protons at relativistic energies up to the protontrapping limit in Jupiter's magnetosphere, which extends to several tens of $\mathrm{GeV}$ (Birmingham 1982).

Our study's implications go beyond the search for JCRPs. The reconstruction of the LEMMS GCR signal has shown that the force-field approximation for GCRs in the heliosphere, expressed through Equations (1) and (2), is also practical and applicable at $\sim 9.6 \mathrm{au}$, a transition region in the heliosphere where ICMEs and CIRs coalesce into (global) merged interaction regions and corotating merged interaction regions (Burlaga \& Ness 1998; Wang \& Richardson 2002; Prise et al. 2015). The approximation also appears to capture dynamics at intermediate timescales (50-100 days), as at 1 au (Usoskin et al. 2015). Furthermore, the LEMMS measurements, being among the few long-term GCR time series at a fixed heliocentric distance other than $1 \mathrm{au}$, are offered for a variety of heliospheric studies, such as GCR transport (Florinski et al. 2003) and calculations of solar wind propagation toward the outer edge of the heliosphere (Krimigis et al. 2013; Witasse et al. 2017). Finally, the relatively well-defined and weakly variable GCR spectrum offers an ideal target for validating and optimizing the in-flight calibration of LEMMS's highestenergy responses (Section 3.2). This is an ongoing process (Roussos et al. 2018b) that will extend the applicability of the LEMMS measurements for a variety of magnetospheric and heliospheric investigations.

We thank Andreas Lagg and Markus Fränz (MPS) for extensive software support and Martha Kusterer and Jon Vandegriff (both JHUAPL) for reducing the MIMI data. Cassini data analysis at MPS was supported by the German Space Agency (DLR) through contracts $50 \mathrm{OH} 1101$ and 50 $\mathrm{OH} 1502$ and by the Max Planck Society. The JHUAPL authors, as well as K.D., were partially supported by the NASA Office of Space Science under task order 003 of contract NAS5-97271 between NASA/GSFC and JHU and subcontract to the Academy of Athens. E.E. thanks the Brazilian Federal Research Foundation agency (CNPq302583/2015-7) for financial support.

\section{Appendix A Non-GCR Proton Contributions to the LEMMS Signal}

Evidence that the LEMMS measurements shown here (Figure 3) are dominated by GCR protons and not particles from Saturn's magnetosphere has been presented in a series of publications, but for completeness, we collectively summarize them here. We also discuss why GCR and Jovian relativistic electrons can be ignored, as these particles have not been considered in the past as sources of a signal in LEMMS near Saturn.

\section{A.1. Contributions from Saturn's Magnetosphere}

Excluding the radiation belts, Saturn's magnetosphere is not an efficient accelerator of protons (e.g., through reconnection), as these have not been observed at energies above $1-2 \mathrm{MeV}$, except at periods when there is a direct input from high fluxes of SEPs during strong ICME events. Such protons drive the formation of transient radiation belts extending to large distances in Saturn's magnetosphere but at proton energies much below those captured by channels E6, P9, and E7 (Roussos et al. 2008, 2018a). The highest-energy protons, which extend to the $\mathrm{GeV}$ range and can trigger the three channels, are seen in Saturn's radiation belts. However, these protons are tightly confined in the inner magnetosphere due to the absorbing effects of Saturn's moons and rings and cannot reach the middle and outer magnetosphere, beyond which all measurements shown here come from (Roussos et al. 2011, 2018b; Kollmann et al. 2013).

The $\mathrm{MeV}$ electrons with energies above 1.6, 3, and $7 \mathrm{MeV}$, which may trigger channels E6, P9, and E7, respectively (Figure 2(b)), are also present in high fluxes in the radiation belts. The electron belts are also limited inward of $\sim 10 R_{\mathrm{s}}$ in Saturn's equatorial magnetosphere (Roussos et al. 2014), regions excluded from our analysis. At distances further from the radiation belts, where data for Figure 3 were acquired, MeV electrons have been observed as products of impulsive, quasiperiodic acceleration events termed QP60, due to their typical hourly periodicity (Palmaerts et al. 2016; Roussos et al. 2016). Most QP60 events have a total duration of $6 \mathrm{hr}$ (while we use daily averages). Those events that extend in the energy range of channels E6 and P9 have even shorter durations $(<2 \mathrm{hr})$ and low count rates that become noticeable only when the GCR proton background is subtracted. No QP60 event has been observed to trigger an electron signal in channel E7.

\section{A.2. Contributions from Jovian and GCR Electrons}

Electrons with energies $<30 \mathrm{MeV}$ are expected have similar flux at 1 and $10 \mathrm{au}$ because their dominant source is Jupiter (Jovian cosmic-ray electrons (JCREs); Strauss et al. 2011; Nndanganeni \& Potgieter 2018). Using the response functions of the three LEMMS channels to MeV electrons $\left(G_{e, i}\right.$; Figure 2(b)) and the properties of the Jovian electron spectrum at Earth (Vogt et al. 2018), we estimate an upper limit of their expected count rate through

$$
R_{\mathrm{JCRE}, i}=4 \pi \int_{T_{\min }}^{100 \mathrm{MeV}} j_{\mathrm{JCRP}}(T) G_{e, i}(T) d T .
$$

The peak contribution is for channel E6 at $\sim 5 \times$ $10^{-3}$ counts $^{-1}$, a negligible amount compared to the average rate of $\sim 2$ counts $\mathrm{s}^{-1}$ due to GCR protons. The primary reason for the low contribution by such electrons in LEMMS is that the estimated electron count rate results from an integration of the electron spectrum over an energy interval of "just" 30-40 MeV, where electron fluxes are most significant. For protons, this integration range is over several $10 \mathrm{~s}$ of $\mathrm{GeV}$, with geometry factors also being larger than the corresponding electron ones. Since E6, P9, and E7 cannot discriminate between $\mathrm{MeV}$ electrons and $>100 \mathrm{MeV}$ protons, they record 
the sum of the two contributions, which is then clearly dominated by GCR protons.

\section{Appendix B \\ Upper Limits of JCRP Count Rates in MIMI/LEMMS}

In order to estimate the upper limits of the JCRP count rates in the three LEMMS channels used in this study, we first assume that the fractional abundance of JCRPs in GCRs is between $1 \%$ and $4.8 \%$ of the average GCR signal at $1 \mathrm{au}$. These percentages are given in Pizzella (2018) for three energy ranges, with the lowest values corresponding to the highest energies. We use these percentages, together with GCR fluxes from Adriani et al. (2013), to describe the upper flux limits of the JCRP spectrum at 1 au as

$$
\begin{aligned}
& j_{\mathrm{JCRP}}(T)\left[\left(m^{2} \mathrm{~s} \mathrm{sr} \mathrm{GeV}^{-1}\right]\right. \\
& =\left\{\begin{array}{l}
22.4 T[\mathrm{GeV}]^{-0.51}, T<1.67 \mathrm{GeV} \\
61.7 T[\mathrm{GeV}]^{-2.51}, T \geqslant 1.67 \mathrm{GeV} .
\end{array}\right.
\end{aligned}
$$

The expected upper limits for the LEMMS rates from JCRPs can then be estimated by integrating the spectrum of Equation (6) over energy, similar to Equation (3):

$$
R_{\mathrm{JCRP}, i}=\frac{4 \pi}{k_{i}} \int_{T_{\min }}^{50 \mathrm{GeV}} j_{\mathrm{JCRP}}(T) G_{i}(T) d T .
$$

Values for $k_{i}$ in Equation (5) are based on the analysis in Section 3.2 and given in Table 1. Application of Equation (5) predicts upper limits of $4 \times 10^{-3}$ counts s$^{-1}$ for channel E7, 0.02 counts $\mathrm{s}^{-1}$ for $\mathrm{P} 9$, and 0.04 counts s$^{-1}$ for E6, values smaller than or comparable to the standard deviation of each channel's residual signal (Table 2). These values can be considered as extreme upper limits, since the 1\%-4.8\% fractional abundance of JCRPs in GCRs is likely overestimated, as discussed in Section 4 and in the context of Figure 7.

\section{ORCID iDs}

Elias Roussos (1) https://orcid.org/0000-0002-5699-0678 Norbert Krupp (ㄴ) https://orcid.org/0000-0003-4683-9533 Konstantinos Dialynas (10 https://orcid.org/0000-00025231-7929

Peter Kollmann (1) https://orcid.org/0000-0002-4274-9760 Christopher Paranicas (10) https://orcid.org/0000-00024391-8255

Ezequiel Echer (ㄴ) https://orcid.org/0000-0002-8351-6779 Stamatios M. Krimigis (10) https://orcid.org/0000-00032781-2386

\section{References}

Adriani, O., Barbarino, G. C., Bazilevskaya, G. A., et al. 2013, ApJ, 765, 91 Adriani, O., Barbarino, G. C., Bazilevskaya, G. A., et al. 2018, ApJL, 852, L28 Anagnostopoulos, G. C., Louri, I., Marhavilas, P., \& Sarris, E. T. 2009, AdSpR, 43, 573

Anglin, J. D., Burrows, J. R., Mu, J. L., \& Wilson, M. D. 1997, JGR, 102, 1 Bazilevskaya, G., Broomhall, A.-M., Elsworth, Y., \& Nakariakov, V. M. 2014, SSRv, 186, 359

Becker, H. N., Alexander, J. W., Adriani, A., et al. 2017, SSRv, 213, 507

Birmingham, T. J. 1982, JGR, 87, 7421

Blasi, P. 2013, A\&ARv, 21, 70

Burlaga, L. F., \& Ness, N. F. 1998, SSRv, 83, 105

Caballero-Lopez, R. A., \& Moraal, H. 2004, JGRA, 109, A01101

Cane, H. V. 2000, SSRv, 93, 55

Dragt, A. J., Austin, M. M., \& White, R. S. 1966, JGR, 71, 1293
Echer, E. 2019, P\&SS, 165, 210

Ferreira, S., Potgieter, M., Heber, B., Fichtner, R., \& Kissmann, R. 2003, AdSpR, 32, 669

Fischer, H. M., Pehlke, E., Wibberenz, G., Lanzerotti, L. J., \& Mihalov, J. D. 1996, Sci, 272, 856

Florinski, V., Zank, G. P., \& Pogorelov, N. V. 2003, JGRA, 108, 1228

Garrard, T. L., Gehrels, N., \& Stone, E. C. 1992, in The Galileo Heavy Element Monitor, ed. C. T. Russell (Dordrecht: Springer Netherlands), 305

Giacalone, J., Drake, J. F., \& Jokipii, J. R. 2012, SSRv, 173, 283

Gieseler, J., \& Heber, B. 2016, A\&A, 589, A32

Gleeson, L. J., \& Axford, W. I. 1968, ApJ, 154, 1011

Hassler, D. M., Zeitlin, C., Wimmer-Schweingruber, R. F., et al. 2014, Sci, 343, 1244797

Heber, B., Fichtner, H., \& Scherer, K. 2006, SSRv, 125, 81

Heber, B., Potgieter, M., Ferreira, S., et al. 2007, P\&SS, 55, 1

Hill, M. E., Kollmann, P., Brown, L., \& McNutt, R., Jr. 2018, in Solar Heliospheric and INterplanetary Environment (SHINE 2018), 98

Jackman, C. M., Forsyth, R. J., \& Dougherty, M. K. 2008, JGRA, 113, A08114

Jokipii, J. 2013, in Cosmic Rays (Washington, DC: American Geophysical Union), 123

Kollmann, P., Roussos, E., Paranicas, C., Krupp, N., \& Haggerty, D. K. 2013, Icar, 222, 323

Kotova, A., Roussos, E., Kollmann, P., Krupp, N., \& Dandouras, I. S. 2019, JGRA, in press (doi:10.1029/2018JA025661)

Krimigis, S. M., Armstrong, T. P., Axford, W. I., et al. 1977, SSRv, 21, 329

Krimigis, S. M., Decker, R. B., Roelof, E. C., et al. 2013, Sci, 341, 144

Krimigis, S. M., Mitchell, D. G., Hamilton, D. C., et al. 2004, SSRv, 114, 233

Krimigis, S. M., Sarris, E. T., \& Armstrong, T. P. 1975, GeoRL, 2, 561

Krimigis, S. M., Sergis, N., Dialynas, K., et al. 2009, P\&SS, 57, 1785

Krivova, N. A., \& Solanki, S. K. 2002, A\&A, 394, 701

Krupp, N., Woch, J., Lagg, A., et al. 2002, GeoRL, 29, 26

Mandal, S., Hegde, M., Samanta, T., et al. 2017, A\&A, 601, A106

Marhavilas, P. K., Anagnostopoulos, G. C., \& Sarris, E. T. 2001, P\&SS, 49, 1031

Mauk, B. H., Fox, N. J., Kanekal, S. G., et al. 2013, SSRv, 179, 3

Mitra, B., Bose, S. K., \& Ganguly, S. R. 1982, NCimL, 33, 9

Mitra, B., Bose, S. K., \& Ganguly, S. R. 1983, ICRC (Bangalore), 3, 182

Nagashima, K., \& Tatsuoka, R. 1984, NCimC, 7, 379

Nndanganeni, R. R., \& Potgieter, M. S. 2018, Ap\&SS, 363, 156

Obridko, V., \& Shelting, B. 2007, AdSpR, 40, 1006

Palmaerts, B., Roussos, E., Krupp, N., et al. 2016, Icar, 271, 1

Picozza, P., Galper, A. M., Castellini, G., et al. 2007, APh, 27, 296

Pizzella, G. 2018, EPJC, 78, 848

Pizzella, G., \& Venditti, G. 1973, ICRC (Denver), 2, 1129

Potgieter, M. S. 2013, LRSP, 10, 3

Potgieter, M. S. 2017, AdSpR, 60, 848

Prise, A. J., Harra, L. K., Matthews, S. A., Arridge, C. S., \& Achilleos, N. 2015, JGRA, 120, 1566

Reames, D. V. 1999, SSRv, 90, 413

Roussos, E., Jackman, C., Thomsen, M., et al. 2018a, Icar, 300, 47

Roussos, E., Kollmann, P., Krupp, N., et al. 2018b, Sci, 362, aat1962

Roussos, E., Krupp, N., Armstrong, T. P., et al. 2008, GeoRL, 35, 22106

Roussos, E., Krupp, N., Mitchell, D. G., et al. 2016, Icar, 263, 101

Roussos, E., Krupp, N., Paranicas, C., et al. 2014, P\&SS, 104, 3

Roussos, E., Krupp, N., Paranicas, C. P., et al. 2011, JGRA, 116, A02217

Selesnick, R. S., Cohen, C. M. S., \& Khurana, K. K. 2001, JGR, 106, 18895

Semkova, J., Koleva, R., Benghin, V., et al. 2018, Icar, 303, 53

Simpson, J. A., Anglin, J. D., Balogh, A., et al. 1992, A\&AS, 92, 365 Spergel, M. S. 1977, Moon, 17, 123

Stilwell, D. E., Davis, W. D., Joyce, R. M., et al. 1979, ITNS, 26, 513

Stone, E. C., Cohen, C. M. S., Cook, W. R., et al. 1998, SSRv, 86, 285

Strauss, R. D., Potgieter, M. S., Büsching, I., \& Kopp, A. 2011, ApJ, 735, 83

Teegarden, B. J., McDonald, F. B., Trainor, J. H., Webber, W. R., \& Roelof, E. C. 1974, JGR, 79, 3615

Usoskin, I. G., Gil, A., Kovaltsov, G. A., Mishev, A. L., \& Mikhailov, V. V. 2017, JGRA, 122, 3875

Usoskin, I. G., Kovaltsov, G. A., Adriani, O., et al. 2015, AdSpR, 55, 2940

Vainio, R., Desorgher, L., Heynderickx, D., et al. 2009, SSRv, 147, 187

Vogt, A., Heber, B., Kopp, A., Potgieter, M. S., \& Strauss, R. D. 2018, A\&A, 613, A28

Wang, C., \& Richardson, J. D. 2002, GeoRL, 29, 1181

Williams, D. J., McEntire, R. W., Jaskulek, S., \& Wilken, B. 1992, SSRv, 60,385

Witasse, O., Sánchez-Cano, B., Mays, M. L., et al. 2017, JGRA, 122, 7865 Zhang, M., McKibben, R. B., Simpson, J. A., et al. 1995, JGR, 100, 19497

Zhang, Y., Sun, W., Feng, X. S., et al. 2008, JGRA, 113, a08106 